\title{
Objetos de aprendizaje y enseñanza bibliotecológica
}

\author{
Learning Objects and Library Science Teaching
}

Miguel Sosa (1), Martha Ibáñez Marmolejo (2) y Verónica Soria Ramírez (3)

(1) CINVESTAV, Sección de Metodología y Teoría de la Ciencia, Av. Instituto Politécnico Nacional \#2508, Col. San Pedro Zacatenco, CP 07360, México, D. F., msosa@cinvestav.mx (2) Centro Universitario de Investigaciones Bibliotecológicas, UNAM. Torre II de Humanidades, Piso 11, Ciudad Universitaria, CP 04510, México, D. F., ibanez@cuib.unam.mx (3) Dirección General de Bibliotecas, UNAM. Edificio de Biblioteca Central s/n Coyoacán, CP 04510, México, D. F., documentos@dgb.unam.mx

\begin{abstract}
Resumen
En el estadio actual del saber humano el alumno no sólo debe adquirir conocimientos para desempeñarse eficientemente en el campo laboral y profesional, sino también competencias y habilidades que le permitan desarrollar todo su potencial intelectual y operativo. Actualmente, las tendencias tecnológicas y pedagógicas para el diseño, desarrollo y entrega de contenidos educativos se orientan hacia los Objetos de Aprendizaje, debido a su enorme potencial de reusabilidad, granularidad, capacidad generativa, adaptabiliad y escalabilidad. En el área bibliotecológica, los Objetos de Aprendizaje pueden constituirse como una herramienta que cubra las necesidades de aprendizaje de manera óptima, puesto que sus características pedagógicas facilitan y propician un aprendizaje significativo.
\end{abstract}

Palabras clave: Aprendizaje significativo. Competencias profesionales. E-Learning. Enseñanza bibliotecológica. Objetos de aprendizaje.

\section{Introducción}

En el mundo actual, la información es el principal insumo para la comunicación y la representación del conocimiento. Su empleo es un factor primordial en los procesos de producción de bienes culturales y materiales, por lo cual su rol es equivalente al de las materias primas y la energía en la producción de bienes materiales.

La presencia de las Tecnologías de Información y Comunicación (TIC) y las necesidades concretas del mercado laboral hacen que en la educación superior, tanto en las aulas como en los procesos de aprendizaje, comiencen a hacerse cuestionamientos relacionados con los métodos empleados hasta ahora. Al mismo tiempo han surgido nuevas oportunidades para el estudiante, las cuales es necesario aprovechar, pues la pretensión es estimular la innovación de las

\begin{abstract}
In the current human knowledge stage, the student must acquire not only skills to perform eficiently in the academic and professional fields, but also skills and abilities enabling him to develop his full intellectual and operational potential. Current technological trends for the design, development and delivery of educational content are geared toward Learning Objects, due to its enormous potential for reusability, granularity, capacity of adaptability and scalability. In the Library Science area, Learning Objects are a very useful tool, since their pedagogical characteristics facilitate and foster significative learning.
\end{abstract}

Keywords: E-Learning. Learning objects. Education in Library Science. Information Technologies and Education. Professional competencies. Significant learning.

prácticas docentes para facilitar los procesos de aprendizaje encaminados a propiciar la reconstrucción de las experiencias e informaciones, con la finalidad de integrar los conocimientos propios, locales, a las exigencias de un mundo global e interconectado. Esto significa cambios en los procesos formativos, donde el alumno se convierte en el protagonista de tales acciones, disponiendo de gran autonomía y control sobre su propio proceso de aprendizaje.

Una nueva alternativa para la formación profesional que tiene la capacidad de ofrecer un amplio acceso y soporte para el aprendizaje continuo, es el denominado E-learning. Sin embargo dicha modalidad afronta algunos obstáculos. Por una parte, requiere un diseño especial tanto de las aplicaciones como de los contenidos para dar respuesta a las demandas de aprendizaje; "además de que no estén realizados bajo una 
clara noción de diseño instruccional, esto hace que dicha modalidad se convierta más en ereading que en e-learning" (Muñoz Arteaga, 2006). Por otra, se requiere una inversión considerable para la creación de cursos en línea.

La creación de contenido reutilizable que pueda ser compartido por varias personas o aplicaciones es una idea que actualmente cobra fuerza e interés por parte de especialistas de varias disciplinas; teniendo como fin diseñar recursos que puedan adaptarse a diferentes modelos educativos, temas y niveles de estudio.

Vale destacar que la calidad de los contenidos para las actividades de e-learning es el elemento más importante que refleja la seriedad y garantiza la imagen de la modalidad. Por ello, los contenidos deberán proporcionar no sólo la explicación de los fenómenos, sino también sus posibles soluciones, aplicaciones y vinculación con los problemas a los que se enfrentan.

La tendencia tecnológica para el diseño, desarrollo y entrega de contenidos educativos, en el momento actual se orienta hacia los Objetos de Aprendizaje (OA) debido a sus múltiples ventajas. Estos están encaminados a la capacitación basada en habilidades y competencias, a fin de que sea posible construir un programa de capacitación exactamente a la medida del perfil del alumno. De este modo, los objetos de aprendizaje tienen un enfoque eminentemente práctico, en el cual es posible desarrollar herramientas pequeñas de instrucción, susceptibles de ser compartidas y reutilizadas en diferentes contextos y que, además, puedan ser combinadas para construir módulos de instrucción mayores.

El trabajo se avoca a describir los conceptos básicos relacionados con la formación por competencias y proponer la aplicación de los objetos de aprendizaje como una herramienta de apoyo para el desarrollo de contenidos con base en el aprendizaje significativo dentro de la disciplina bibliotecológica.

\section{Competencias}

La educación y el conocimiento constituyen indudablemente las bases de nuestra sociedad. Hoy más que nunca, el conocimiento y su generación son determinantes y decisivos para el desarrollo. Frente a esta realidad, el papel de las competencias asume una nueva dimensión.

La OCDE señala que en el contexto de la sociedad que conocemos, los individuos necesitan manejar competencias que les permitan adaptarse a un mundo complejo, cambiante e interdependiente donde:
- La tecnología cambia rápida y continuamente; por lo tanto, más que dominio, se requiere desarrollar la adaptabilidad a ella.

- La sociedad es cada vez más diversa y compartimentada; por lo tanto, se requieren relaciones con personas muy diferentes unas de otras.

- La globalización está creando nuevas formas de interdependencia que producen efectos tanto a nivel local como nacional y mundial.

Los desafíos que enfrenta la educación superior están produciendo cambios en el perfil del estudiante y en la modalidad de interacción docentealumno. Ahora, los procesos educativos se caracterizan por la intencionalidad educativa, la planificación y el desarrollo sistemático de un conjunto de prácticas educativas específicas que tienen como finalidad la facilitación de la construcción de conocimiento por parte del estudiante, además de proporcionarle los elementos necesarios para que pueda desarrollar competencias profesionales en el mercado laboral y posea las herramientas suficientes para mantener sus procesos de aprendizaje a lo largo de la vida; de esta forma (Valdez Coiro, 2006):

[...] los tres pilares en los cuales descansa la competitividad son la tecnología, los sistemas de trabajo y la formación de recursos humanos que puedan utilizarla y adaptarse a los nuevos sistemas de trabajo en forma oportuna para dar respuesta a la demanda del ámbito laboral.

Las competencias entraron a la educación por factores externos, tales como competencia empresarial, globalización y la internacionalización de la economía. En la década de 1970, cuando las empresas y organizaciones hacían la selección de su personal mediante test de inteligencia y exámenes de conocimiento y creían que las personas con mayor coeficiente intelectual y con mejores notas podían ser los profesionales más exitosos en las organizaciones, David Mc Clelland (1) inició una investigación sobre el porqué unos empleados tenían más éxito que otros en el trabajo. Por medio del método de incidentes críticos identificó qué cualidades o aptitudes hacían que un trabajador lograra resultados excepcionales. Dicho estudio le llevó a la conclusión que no había correlación entre los profesionales de mayor coeficiente intelectual y el éxito, sino que este dependía de otras características personales como aptitudes y motivaciones. Como resultado de dichas investigaciones, Mc Clelland acuñó en 1973 el concepto de competencias, definiéndolas como las características subyacentes de la personalidad que tienen una relación causal con criterios referen- 
ciados con el desarrollo efectivo o superior en un trabajo o situación.

Becker (2), economista ganador del Premio Nobel en 1992, comenzó a estudiar las sociedades del conocimiento varios años antes y concluyó que su mayor tesoro era el capital humano que éstas poseían, esto es, el conocimiento y las habilidades que forman parte de las personas, su salud y la calidad de sus hábitos de trabajo, llegando a sostener la importancia del capital humano para la productividad de las economías modernas, ya que esta productividad se basa en la creación, difusión y utilización del saber.

Mucho se ha dicho sobre las competencias, pero todavía hay grandes vacíos y desacuerdos sobre su definición. La conceptualización del término depende "del enfoque respecto de la gestión del talento humano, de la valoración que se otorgue, al trabajador y al trabajo en la acumulación de valor" (Tobón, 2004).

La perspectiva de las competencias obliga a comprender mejor los propios procesos de aprendizaje, a fin de utilizarlos de manera óptima. De ahí el interés que hoy tiene, para elevar la efectividad de la formación, conocer en profundidad cómo aprenden las personas y las organizaciones.

Las competencias son una compleja estructura de atributos que algunas personas dominan mejor que otras, donde se combinan conocimiento, actitudes, valores y habilidades necesarios para un mejor desempeño en situaciones determinadas. El hecho de identificar competencias puede ser útil para familiarizar a la institución con algunas de sus principales fortalezas y debilidades, así como para tener acceso a ideas que pueden llegar a constituir hitos apropiados para reforzar el proceso de mejoramiento permanente.

Las competencias son el conjunto de conocimientos, procedimientos y actitudes combinados, coordinados e integrados en la acción, adquiridos a través de la experiencia (formativa y no formativa) que permite al individuo resolver problemas específicos de forma autónoma y flexible en contextos singulares (Tejeda, 2001).

Las competencias, además de conocimientos técnicos o metodológicos incluyen también aspectos relacionales y sociales, los cuales son descritos en el trabajo de Bunk (1994). Para este autor, una persona competente es la que dispone de los conocimientos, destrezas y aptitudes necesarios para ejercer una profesión, puede resolver los problemas profesionales de forma autónoma y flexible y está capacitado para colaborar en su entorno profesional y en la organización del trabajo. En la figura 1 se ilustran tales afirmaciones.

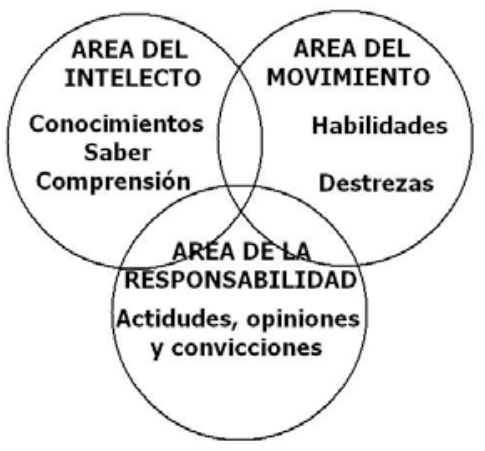

Figura 1. Bunk, 1994.

Ante este panorama, las competencias se han convertido en un nuevo paradigma de la formación profesional en el ámbito internacional.

Señala Valdez (2006), que la formación basada en competencias reconoce el aprendizaje adquirido por la experiencia o por cualquier otro medio, optimizando el tiempo y el esfuerzo de los participantes. Algunos de los elementos distintivos de dicha propuesta son:

- Considera referentes laborales o académicos.

- Es de tipo modular para fomentar la flexibilidad; y, con ello, algunos beneficios, como son el tránsito de rutas curriculares o aprendizajes parciales dentro de una carrera, entre otros.

- Requiere validez, confiabilidad y objetividad en la evaluación, la cual privilegia desempeños y productos por sobre el conocimiento per se.

- Se centra en el aprendizaje, privilegiando la actividad de los alumnos sobre la de los docentes, al atender mediante los recursos multimedia diversos estilos de aprendizaje por medio de estrategias didácticas enlazadas con la aplicación de las competencias a ámbitos laborales o reales.

- Los comportamientos que desarrolla la educación por competencias tienen la característica de ser molares, es decir, complejos, sintetizando conocimientos, habilidades, destrezas $y$ actitudes en ellos, y no desarrollando de manera atomística capacidades parciales.

Desde la perspectiva de las competencias, el interés de los procesos formativos ha de ser, fundamentalmente, el desarrollar los comportamientos asociados a aquéllas. Por ello la formación no puede ser una simple superposición de conocimientos, sino la transformación de unos 
determinados comportamientos ligados a las ocupaciones (Lopez, 2002).

\section{Educación bibliotecológica}

En los años recientes hemos sido testigos de cambios en la educación superior relativos a estrategias, modelos y métodos que pretenden en su conjunto dar soluciones a las necesidades que plantea la sociedad actual; desde esta misma óptica la educación bibliotecológica también ha cambiado, pues los esfuerzos por organizar los aspectos relacionados con la enseñanza han originado investigaciones que permiten el desarrollo de metodologías más flexibles en los logros del aprendizaje.

Así, en la disciplina bibliotecológica, Morales refiere que es necesario "buscar los caminos y métodos para que los decires de los siglos estén a disposición pública y abierta de la sociedad, e ir enriqueciendo las nuevas demandas de la sociedad que obligan al bibliotecólogo a renovar sus compromisos con la sociedad y sus usuarios" (Morales, 2003).

Por lo tanto, la educación bibliotecológica debe adecuarse tomando en cuenta las condiciones actuales y las necesidades concretas del país. En México se imparte la disciplina en varias instituciones (3). Las escuelas de bibliotecología están llamadas a potenciar la formación profesional, en consideración a los cambios vigentes en los escenarios actuales y sus posibles repercusiones en los escenarios futuros.

La intención del enfoque de competencias en la disciplina, es tratar de abrir espacios dentro de la enseñanza técnica, al estudio de la teoría bibliotecológica y sus fundamentos, se pretende esencialmente superar el enfoque de enseñanza técnica por un enfoque de disciplina teórica (Ríos, 2007).

\subsection{Competencias en bibliotecología}

En los últimos años los programas de estudio en la disciplina han experimentado diversas transformaciones, considerando aspectos para la mejora de conocimientos, habilidades y competencias de los alumnos hacia su formación profesional.

El objetivo principal es formar un nuevo profesional de la información que responda adecuadamente a la realidad del presente siglo. Para ello es necesario incorporar elementos de innovación que contribuyan a replantear modelos y estilos de gestión tradicionales que, si bien respondieron a otros momentos históricos particulares, en la actualidad han sido superados y resultan obsoletos ante el avasallante progreso científico y tecnológico que plantea nuevas y complejas necesidades informacionales y cognitivas (Pirela, 2006).

El nuevo escenario académico requiere de profesionales de la información con un perfil amplio que, conducidos por la acción, determinen el comportamiento que los haga cumplir con su misión, transformar la visión social de este profesional y elevar su influencia en la cultura nacional, regional y universal.

La noción de competencia en el ámbito bibliotecológico debe ser entendida como aquellos saberes de ejecución dentro de las teorías de cognición, porque todo conocer se traduce en un saber, de lo cual se puede interpretar que las competencias y el saber son recíprocos: saber pensar, saber desempeñar, saber interpretar, saber actuar en diferentes escenarios. En la figura 2 se pueden apreciar de manera gráfica las afirmaciones precedentes.

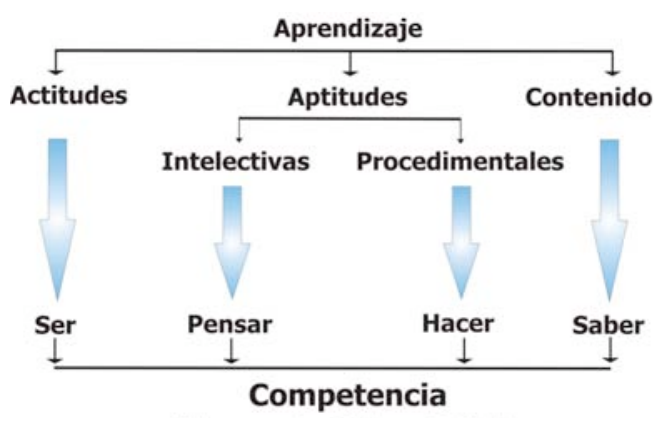

Figura 2. Salas, 2005.

Los distintos tipos de saber permiten comprender la exigencia de una enseñanza basada en competencias en la cual los aprendizajes tienden a ser comprendidos en forma diferente (Zambrano, 2006)

En Bibliotecología educar en competencias es educar en conocimientos, es otro camino para educar, implica rediseñar los contenidos orientándolos hacia una formación en sentido amplio, estableciendo las bases y la profundización disciplinar suficientes para garantizar tanto un desarrollo personal e intelectual como una idoneidad en sintonía con las demandas del mercado laboral.

La Special Libraries Association (SLA) en su estudio Competencies of Information Professionals of the 21ts Century, destaca que los bibliotecarios especiales necesitan de dos clases principales de competencias: las competencias profesionales (conocimientos especiales en recursos de información, acceso a la información, tecnología, manejo de la información y la habilidad para el manejo de la misma) y las 
competencias personales - habilidades, actitudes, aptitudes y valores que permitan a los bibliotecarios trabajar de forma eficiente y enfocarse en el aprendizaje continuo (SLA, 1996).

Otra postura sobre las competencias profesionales en el área bibliotecológica se encuentra plasmada en el Euroreferencial en Información y Documentación, cuyas líneas guía se han enfocado a desarrollar un sistema integral de evaluación que permita demostrar la calidad del trabajo que desarrollan los profesionales de la información y documentación, valorar la experiencia con que cuentan, avalar la solidez de los conocimientos que poseen, mejorar la competitividad ante el mercado internacional y fortalecer la profesión. Desde esta óptica, las competencias se jerarquizan en cuatro niveles de especialización laboral:

- Nivel 1: Sensibilización. Uso de las herramientas en un nivel elemental.

- Nivel 2: Práctico. Profesional que domina las herramientas básicas realizando tareas específicas o repetitivas, colabora con especialistas de contenido.

- Nivel 3: De ejecución. Conoce todas las técnicas profesionales, las expone y amplía y emite juicios.

- Nivel 4: De desarrollo. Domina la metodología, puede desarrollar nuevos sistemas, se encarga de la gestión más allá de la unidad de información propiamente dicha.

El concepto de competencias está ligado a las experiencias particulares, donde el docente las relaciona con la capacidad, los logros y la realización, mientras que para el mercado laboral se asocian con un excelente desempeño en el trabajo, alta producción y profesionalismo. Por su parte se espera que el especialista formado con este enfoque desarrolle capacidades de aprendizaje a lo largo de la vida que le permitan mantener altos estándares de calidad en su desempeño profesional.

En síntesis, podemos afirmar que se requiere de un amplio proceso de discusión para llegar a acuerdos sobre los contenidos y competencias del profesional de la información, y sobre las exigencias de los niveles de calidad con los cuales deben regirse las instituciones responsables de la formación de los recursos humanos insertos en la Sociedad del Conocimiento.

Como señala Benítez (2002) "las competencias deben desarrollar profesionales enriquecidos para asimilar conocimientos teórico-prácticos, integrarlos y/o vincularlos con otros campos del saber, además de aplicar métodos de investigación para generar su propio conocimiento".

\section{E-learning}

Raffaele Simone (2001) plantea la existencia de tres fases que se manifiestan alrededor de la historia sobre el modo en que se forman los conocimientos de la humanidad y se alimenta el patrimonio del saber. Este paso de una a otra de las grandes fases de la historia del conocimiento ha sido producido por dos tipos de fenómenos distintos: uno técnico y otro mental. El fenómeno técnico consiste en el hecho de que se inventan continuamente "instrumentos" materiales nuevos vinculados con el conocimiento: primero, el estilo y la pluma; después, la imprenta; en la actualidad, la computadora y los media. El mental, en cambio, está constituido por el paso, primero, de la oralidad a la escritura; y, segundo, de la lectura a la visión y a la escucha. La irrupción de las TIC e Internet están modificando las aulas y el sistema de aprendizaje, obligando a que los distintos actores participen en el sistema educativo adaptándolo a los nuevos tiempos.

En el proceso de aprendizaje el e-learning o teleformación, modifica la visión y el rol de los agentes involucrados porque se establece una combinación entre la aplicación de Internet y las TIC para facilitar los procesos de gestión del conocimiento que permiten una modificación en la enseñanza así como del modo en cómo se aprende, propiciando de igual manera un entorno más democrático, abierto y participativo, lo cual constituye una muestra más de que nos hallamos involucrados en una Sociedad del Conocimiento.

Alexandra Draxler (2004) manifiesta las diferencias entre el modelo educativo tradicional y el elearning, las cuales radican en cuatro elementos:

- Adquisición de información: En e-learning dicha adquisición se encuentra directamente controlada por la persona que adquiere los conocimientos.

- Transformación de la información en conocimiento: El e-learning ofrece la oportunidad de sumergirnos en una multitud de fuentes de información que debemos saber seleccionar, clasificar y valorar.

- Mediación: El sistema de enseñanza-aprendiaje (e-a) virtual no implica a priori la necesidad de una mediación humana.

- Validación: Los modelos educativos tradicionales y el e-learning siguen compartiendo 
prácticamente los mismos problemas y las mismas técnicas.

Ante la carencia de consenso, y la denominación unívoca del e-learning en el escenario mundial se perciben múltiples miradas sobre las perspectivas y enfoques. Repasemos brevemente algunos. Para la E-Learning Europe " es el uso de las nuevas tecnologías multimedia y de Internet para mejorar la calidad del aprendizaje mediante el acceso a recursos y servicios, y a colaboraciones e intercambios a larga distancia". Por su parte, el Grupo de E-Learning de la NSCA considera que "es la adquisición y el uso de conocimiento distribuido y facilitado básicamente por medios electrónicos. El e-learning puede adquirir el formato de curso, de módulo o de objetos de aprendizaje menores y puede incorporar un acceso síncrono o asíncrono y distribuirse geográficamente con una variedad de tiempo limitado".

Garrison \& Anderson (2005) reafirman que en términos generales, e-learning es un sistema de aprendizaje en red y en línea que tiene lugar en un contexto formal y que pone en juego toda una serie de tecnologías multimedia, el cual puede hacer posible el aprendizaje asincrónico y conjunto.

El e-learning se percibe como un fenómeno que produce una revolución tecnológica en el ámbito de la educación, dado que modifica la educación tradicional más allá de los cánones tradicionales de trasmisión y percepción del conocimiento. En está modalidad, se enfatiza, por un lado, el uso de la tecnología; y, por otra, los procesos de aprendizaje. Sus características se resumen en:

- Separación física entre el profesor y el alumno, ubicados en sitios geográficamente distintos.

- Uso de soportes tecnológicos para asegurar la comunicación entre profesor y alumno.

- Existencia de comunicación bilateral (sincrónico y asincrónica) de manera que se establezca retroalimentación entre docentes y dicentes.

- La formación personalizada está garantizada.

Con lo anterior, se puede recapitular que elearning es un sistema educativo que utiliza todos los recursos de las TIC para crear un ambiente propicio para el proceso de aprendizaje; dicho proceso tiene un cierto nivel de complejidad que depende de la adquisición de conocimientos subordinados, poniendo de relieve el carácter jerárquico y las exigencias de la instrucción adecuada. De esta manera, sólo se produce aprendizaje en un nivel jerárquicamen- te superior cuando se han adquirido los niveles inferiores (Gagné, 1989).

Se apoya en los llamados entornos virtuales de aprendizaje, que facilitan las actividades educativas a través de Internet/Intranet, cuya finalidad es reproducir las condiciones de una clase presencial y proporcionar herramientas de comunicación e interacción del alumno con el profesor; de modo que la comunicación entre el maestro y el alumno queda diferida en el tiempo y el espacio, por lo que se pueden presentar dos modalidades de aprendizaje: en tiempo real o sincrónico y en tiempo diferido o asincrónico.

El e-learning está comenzando a cambiar las prácticas tradicionales educativas al presentar una manera flexible y poderosa mediante la cual los individuos se apropian de nuevos conocimientos y destrezas con el apoyo de las TIC. Ello permite, diseminar y tener acceso a información multimedia, hacer uso de simuladores, al tiempo que permite la interacción y colaboración con discentes que pueden estar dispersos alrededor del mundo.

En el aprendizaje, se abre una serie de posibilidades que ayudan a la adquisición del conocimiento, la cual se puede transformar, modificar o cambiar. Esa transformación se produce en el ser humano, y debe estar orientada hacia la aplicación del conocimiento de acuerdo al desarrollo de los objetivos específicos y de las necesidades que tengan por cubrir; es decir, se convierte en parte de la vida. De ahí que el proceso de aprendizaje deba ser placentero, atractivo y satisfactorio tanto si es un aprendizaje para la actividad profesional, como para la diversión o para la vida misma.

La calidad de los contenidos para las actividades de e-learning es un elemento importante que refleja la seriedad y garantiza la imagen de la modalidad. Es decir, la construcción de materiales didácticos que apoyan el proceso educativo es una de las tareas con mayor prioridad, porque en esta modalidad resultan ser la parte medular del proceso de aprendizaje. Los contenidos deberán proporcionar no sólo la explicación de los fenómenos, sino también sus posibles soluciones, aplicaciones y vinculación con los problemas que se enfrentan (Gagné, 1989):

La importancia de esta estructura jerarquizada reside en la posibilidad de estadios de transferencia de aprendizaje, es decir detectar en qué medida el dominio de las capacidades anteriores permite asegurar el aprendizaje de las habilidades posteriores y hasta qué punto aquellas se transfieren a estas últimas. Así el conocimiento de la división depende del conocimiento de la multiplicación y ésta del conocimiento de la suma. 
El desarrollo de contenidos educativos tiene implicaciones de costo y tiempo que en un mercado competitivo resulta inevitable aminorar. Además, el compartir cursos completos es difícil y resulta ineficiente debido a que las necesidades y objetivos de aprendizaje varían de acuerdo a cada institución y a cada persona.

Una posible solución a esta problemática, surgida del entorno tecnológico y respaldada por aspectos pedagógicos y de diseño instruccional la constituye la tendencia tecnológica enfocada al diseño, desarrollo y entrega de contenidos educativos basada en los denominados Objetos de Aprendizaje.

\section{Objetos de Aprendizaje}

La existencia actual de recursos y materiales didácticos permite pensar en un óptimo aprovechamiento de los mismos para el apoyo de procesos de aprendizaje, aplicándolos en cursos de capacitación y actualización, entre otras posibles aplicaciones; incluso es factible disgregarlos para producir nuevos materiales. Sin embargo, una de las limitantes que suele presentarse al anhelar disponer de estos recursos es el formato en el que se encuentran publicados; puesto que la obsolescencia de la tecnología con que fueron creados, aunada a la falta de modularidad y la ausencia de etiquetas para saber qué son y a quién pertenecen, dificultan su reutilización en un entorno puramente digital. Para permitir la reutilización y el uso eficiente de dichos recursos, es necesaria la transformación de éstos en Objetos de Aprendizaje (OA).

El paradigma de los OA es una búsqueda profunda de la evolución hacia la Sociedad del Conocimiento. Su uso propicia mayor flexibilidad en el desarrollo de sistemas de aprendizaje adaptables dentro y fuera de los sistemas escolarizados. Dicha situación produce una enorme demanda de recursos basados en la web, para trabajar y aprender considerando la reusabilidad y la interoperabilidad de materiales de información digitales.

Plantea Chan (2002) que la noción de OA sostiene una fuerte carga de debate epistémico y que es necesario reconocer diferentes modos de entender la relación entre el sujeto y el objeto (s-o). Por lo cual, la aproximación a los OA desde una reflexión epistemológica permite identificar las diferentes posibilidades de relación del so, derivando enfoques teóricos y metodológicos diversos para el diseño y uso de la herramienta en educación. Por otro lado, el diseño de los OA ha sido utilizado en el área de ciencias computacionales pero que se ha difundido en menor medida en la teoría instruccional. Ella misma afirma que los OA pueden ubicarse dentro de un esquema, a fin de identificar sus posibilidades e implicaciones, las cuales están divididos en cuatro aspectos, a saber: conocimiento, currículum, tecnologías educativas y procesos de enseñanza y aprendizaje.

En la literatura inglesa, el término se conoce como learning object (LO), así como también reusable learning objects (RLO). Ciertos autores españoles lo utilizan como contenidos educativos digitales (CED) o contenidos didácticos. En la literatura mexicana los términos usados son objetos de aprendizaje (OA) y objetos de contenido. El término OA representa una combinación del concepto aprendizaje y el paradigma de objeto-orientado con base en las ciencias de la computación. Los dos conceptos son importantes desde la aplicación tradicional del objetoorientado, lo cual tiene dos perspectivas:

(1) Un objeto tradicional en el mundo de las ciencias de la computación es una autodescripción. En otras palabras, contienen toda la información acerca de sí mismo, por lo tanto pueden ser localizados en cualquier tiempo y sus capacidades pueden ser leídas por quien este interesado en usar el objeto.

(2) Un objeto único puede ser usado en diversos lugares, lo cual evita la necesidad para duplicar las capacidades del objeto en todos los lugares.

Santacruz Valencia (2005) destaca el visionario trabajo de Gerard Shaping the mind: computers in education de 1969, donde se mencionan por primera vez a unas "unidades curriculares que pueden hacerse más pequeñas y combinarse, como piezas de LEGO". Tres décadas más tarde, esa idea comienza a materializarse a través del concepto de objetos de aprendizaje, convirtiéndose de facto en una de las innovaciones tecnológicas más influyentes en el campo de la educación.

En 1991, Merrill, Li \& Jones, en su Instructional transaction theory: an introduction escribian sobre la necesidad de disponer de "unidades de conocimiento" que sirvieran para el aprendizaje, al decir que "...los alumnos pueden sólo manejar una cantidad limitada de información a la vez, esto hace necesario la secuenciación de las unidades del conocimiento".

Existen dos analogías para entender mejor la idea de Objetos de Aprendizaje:

a) La primera es la del LEGO, el juego de construcción a partir de distintas piezas pequeñas que, combinadas entre sí, dan lugar a objetos más grandes (como naves espaciales, castillos, tractores, etc). La idea principal es crear pequeñas piezas de instrucción que se puedan en- 
samblar en una estructura instruccional más grande y reutilizarse en otras estructuras instruccionales (Wiley, 2001).

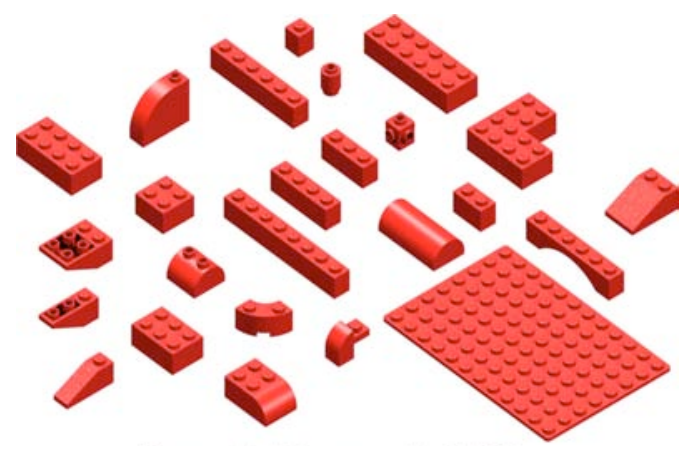

Figura 3. Bloques de LEGO.

Sin embargo, esta primera idea deja de lado algunas características necesarias de contemplar para el correcto uso de los objetos de aprendizaje, ya que el "LEGO" tiene tres particularidades intrínsecas que desvirtúan la idea de Ios OA: cualquier bloque es combinable con otro bloque LEGO; los bloques se pueden combinar en la forma que uno desee; y, finalmente, los bloques son tan divertidos que hasta un niño puede unirlos.

Si la combinación falla, no produce nada más instructivo de lo que los bloques de LEGO enseñan (Wiley, 2001). Es decir, los OA, a diferencia de las piezas de LEGO, deben tener un contexto mínimo, desde donde se haga la lectura (intereses del que enseña, disciplina, punto de vista, etc.) en el que éstos se puedan combinar para que generen un conocimiento productivo.

Para una mejor comprensión del paradigma de los OA, se ha hecho una analogía con la teoría del átomo, la cual se describe a continuación.

b) Un átomo es una pequeña partícula que se puede combinar y recombinar con otros átomos para formar cosas más grandes, pero no todo átomo es combinable con cualquier otro átomo. Los átomos sólo se pueden juntar en ciertas estructuras de acuerdo con su propia naturaleza interna. Finalmente, se requiere cierto entrenamiento para unirlos: no cualquiera puede hacerlo. Aplicando esto a los OA, se deduce que unidades más pequeñas se pueden combinar en estructuras permitidas con cierto número de objetos. Los OA deben ser contextualizados internamente en cierto grado, el mismo que posibilita su combinación con un conjunto cerrado - relacionado-de objetos.

Los OA pueden ser presentados en muchas formas, desde imágenes fijas, solas o acompa- ñadas con texto, clips de video, hipertextos, o combinaciones de éstos. Deben estar clasificados de tal manera que a un usuario le permitan hacer su mapa curricular como mejor le convenga. De esta idea se desprende el criterio de que cada concepto o tema de clase es una unidad, es la parte mínima de información para explicar un concepto; y que éste, a su vez, conjuntado con otros conceptos desarrollan un gran tema; y que, dependiendo de cómo se construya la currícula, por ejemplo, puede llegar a conformar una materia.

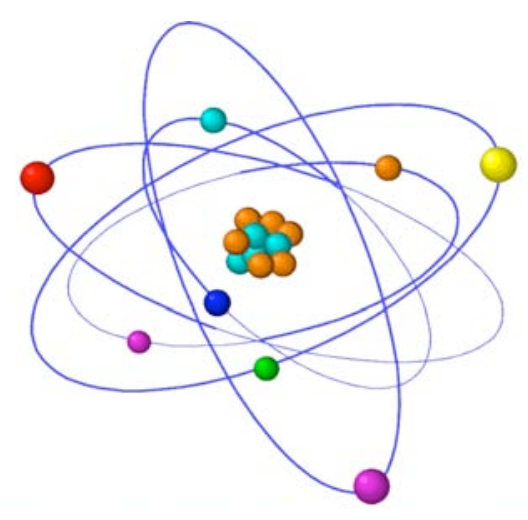

Figura 4. Representación de un átomo.

\subsection{Características}

Banks (2001) en su definición de OA destaca dos características esenciales en el concepto, de un lado la reusabilidad y del otro, la coherencia. En relación a la coherencia identifican tres posibles escenarios:

(a) Reusabilidad por el alumno: Se advierte el empleo de los OA cuanto el estudiante comienza el proceso de formación. Se considera que un $\mathrm{OA}$ es reutilizable por el estudiante cuando el objeto de aprendizaje se encuentra disponible y es fácilmente accesible para él. De esta manera, cada estudiante puede elaborar o construir su propio paquete de objetos atendiendo a sus intereses personales y haciendo posible, de esta forma, que sus metas de aprendizaje queden cubiertas.

(b) Reusabilidad por los proveedores de aprendizaje: Hace referencia al diseño y a la planificación de aquello que se pretende enseñar. Banks expone la posibilidad de elaborar cursos por medio de un repositorio de OA. El proveedor de aprendizaje parte inicialmente de las necesidades formativas de un grupo de estudiantes y desde ellas recupera del repositorio aquellos criterios de formación que se ajustan a su grupo de alumnos. De forma rápida, y económica, se produce un curso que llega a ser un proyecto 
completo de enseñanza-aprendizaje ajustado a las necesidades particulares del grupo de trabajo.

(c) Creación por el principiante: Aprendizaje adquirido por el estudiante, que lo demuestra a través de su propia construcción. Finalmente, también se habla de reusabilidad cuando el estudiante, una vez que ha hecho su propio paquete de $O A$, genera uno diferente, propio y particular. Podríamos decir que es aquí donde la palabra aprendizaje asume su propio significado en tanto que este objeto es, en sentido estricto, el producto del aprendizaje del estudiante. En este momento el estudiante es visto como un participante activo en la construcción del conocimiento, siendo él quien lleva a cabo su propia construcción y la aporta a la comunidad de aprendizaje.

En los tres escenarios anteriores se reconoce la importancia de la coherencia, ya que para ser reutilizados conviene que estén dotados de sentido, dando la sensación de que algo pueda, sea o vaya a ser aprendido. Es por este motivo por el que el autor utiliza la palabra "identificables" puesto que si no hay forma de identificar o describir lo que debería ser aprendido difícilmente podría sugerirse el ofrecer "piezas" coherentes de aprendizaje.

Las características más relevantes que permiten una identificación específica de los OA son:

- Reutilización de contextos o reusabilidad: Los componentes informativos pueden utilizarse las veces que se requiera, en diferentes contextos y de manera simultánea para varios contextos de aprendizaje.

- Interoperabilidad de contenido: Cuentan con metadatos lo cual ofrece la capacidad de moverse de una plataforma a otra, es decir flexibilidad para utilizar los componentes desarrollados en un lugar con herramientas o plataformas localizadas en otro lugar y con herramientas y plataformas diferentes.

- Accesibilidad: Acceso a los componentes desde cualquier lugar y distribuirlos a otros lugares. Debe ser etiquetado para permitir ser almacenado y referenciado.

- Durabilidad: Resistencia a los cambios sin necesidad de rediseñar.

- Granularización del conocimiento: El conocimiento es divido en formas mínimas, es decir la división de contenidos en unidades lógicas de conocimiento como programas, cursos, sesiones o subsesiones y tener acceso de manera flexible, así como compartir y modificar los contenidos en nuevas versiones.
- Independencia y autonomía: Con respecto a los sistemas desde los que fueron creados y con sentido propio.

- Flexibilidad, versatilidad y funcionalidad: Con elasticidad para combinarse en muy diversas propuestas de áreas del saber diferentes.

Ahora bien, además de desarrollar conceptos descriptivos o explicativos, otras características que definen a un $O A$ son que deben contar con un objetivo, una actividad de aprendizaje y una evaluación del contenido. El OA será eficaz en tanto el concepto quede claro independientemente de lo aprendido antes o después. Adquirirá una dimensión distinta y más relevante si es aprendido en un contexto o marco específico, donde cada usuario va conformando su esquema - bitácora- de aprendizaje de acuerdo al contexto que se requiere desarrollar.

\subsection{Estándares}

Por otro lado, los OA necesitan criterios de estandarización con el fin de hacer posible los intercambios, migraciones y uniones de objetos entre repositorios y plataformas distintas. Si los objetos cumplen con determinados estándares sus posibilidades aumentan al permitir combinarlos, ensamblarlos, agruparlos, catalogarlos, entre otros aspectos.

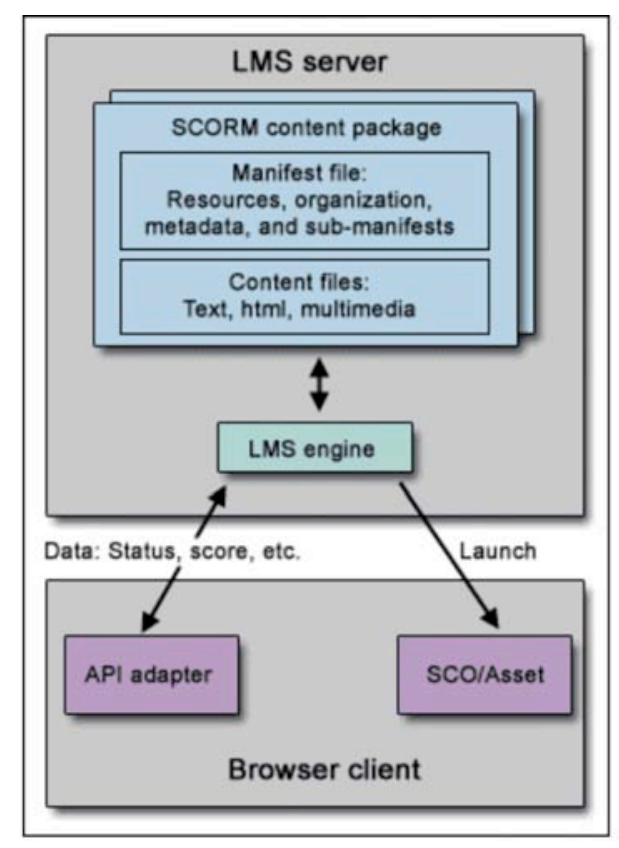

Figura 5. Manifiesto de SCRM

(Fuente: http://builder.com.com).

Como menciona Zapata (2005) los estándares son protocolos que contienen las especificaciones que permitirán dotar de flexibilidad a las 
propuestas de e-learning para su transferencia a los objetos de aprendizaje, tanto en el formato de los materiales y su estructura, como en la conformación de las infraestructuras (herramientas informáticas y telemáticas).

Existen varios estándares para los OA, como el generado en 1996 por el IEEE (Institute of Electrical and Electronics Engineers), el cual es considerado como tutor inteligente en la web. Esta institución desarrolló, además, la arquitectura Learning Technology Standards Committee (LTSC), que provee un marco de trabajo, el cual promueve la reusabilidad, interoperabilidad y portabilidad de sistemas de aprendizaje.

Otro de los estándares es el modelo tecnológico Sharable Content Object Referente Model (SCORM), el cual esta orientado al diseño de objetos de contenidos compartibles. Es una iniciativa de e-learning que plantea una estrategia de reutilización de OA independientes que guardan el conocimiento con la finalidad de soportar un aprendizaje distribuido a partir de los propios objetos. Los criterios de SCORM se agrupan en tres categorías: 1) los que se refieren a empaquetamiento de materiales; 2) los que se refieren a la ejecución de las comunicaciones; y 3 ) los metadatos del curso.

\section{Objetos de Aprendizaje y Educación Bibliotecológica}

En el entendido de que la tecnología es una invaluable herramienta de apoyo para todos los ámbitos del quehacer humano, la aplicación de la misma en el ámbito estrictamente educativo es una oportunidad que debe explorarse a fondo con el fin de obtener el máximo provecho, al tiempo que se puede llegar al mayor número de personas con una inversión y esfuerzo relativamente bajos.

En nuestros días, la Bibliotecología se enfrenta a uno de los cambios más trascendentales en su historia. Somos testigos de una revolución en los medios utilizados para generar, distribuir y utilizar la información, impactando directamente en la práctica profesional y por ende en la formación de los recursos humanos que puedan desenvolverse exitosamente en este nuevo medio.

En palabras de Garduño (2006) es notorio que los fenómenos generados por la innovación tecnológica plantean nuevos retos a la bibliotecología debido a que han transformado modelos y procesos educativos, modificando las formas de comunicación académica y las formas de interacción y comunicación entre los diversos actores del proceso educativo. Asimismo las redes de telecomunicación facilitan cada vez más el acceso a la información en un entorno global; su alta velocidad reduce los límites de fronteras, espacio y tiempo y facilita la recuperación de información requerida en procesos de aprendizaje.

En la educación bibliotecológica es factible hacer uso combinado de las bondades de los objetos de aprendizaje y la formación por competencias, cuya amalgamación podría ofrecer como producto final una plataforma de capacitación y desarrollo de habilidades que permitiese al estudiante aprender de forma modular muchas de las tareas y quehaceres necesarios para su desempeño profesional.

De esta forma, la educación y los programas de formación en la disciplina deben tener la previsión de preparar profesionales que puedan asumir un papel pro-activo en relación a las nuevas tecnologías y a la explosión de la información. Los contenidos de las asignaturas deben concentrarse en desarrollar conocimientos, habilidades y herramientas que se correspondan con las cuatro áreas básicas identificadas: creación, colección, comunicación y consolidación (Karisiddappa, 2004).

En este marco de referencia, el diseño de OA es una propuesta para la disciplina, pues pretende que el alumno sea capaz de desarrollar sus habilidades intelectuales, las cuales podrán estimularse a través de un conjunto de tareas, y la realización de estas permitirá la adquisición y el dominio de los contenidos correspondientes.

Line (1998) considera que está suficientemente claro que los bibliotecarios de hoy deben tener un conjunto de conocimientos y saberes de diferentes dominios que estén aplicados a su actividad particular, pero que, todos ellos, no constituyen un corpus que tenga lugar en el seno de una única disciplina: sólo una pequeña -aunque esencial parte- concierne específicamente a la biblioteconomía, de ahí que puede decirse que los bibliotecarios deben tener un conjunto de competencias pertenecientes a distintas disciplinas, lo que produce una cierta diversificación de la profesión.

La educación bibliotecológica puede auxiliarse de un esquema de objetos de aprendizaje diseñados con el modelo de la formación por competencias, los que a su vez formen parte de un esquema de aprendizaje a distancia, todos los cuales se encuentren soportados en la plataforma de las distintas TIC, que serán las que permitan que dicho conocimiento llegue a sus destinatarios finales. 
Vale la pena mencionar que el diseño curricular, es el soporte teórico, tanto de los objetos de aprendizaje como del esquema de formación por competencias, los cuales deben estar basados en un modelo constructivista, toda vez que dicha propuesta pedagógico-filosófica permitiría fomentar una visión holística de la formación perseguida.

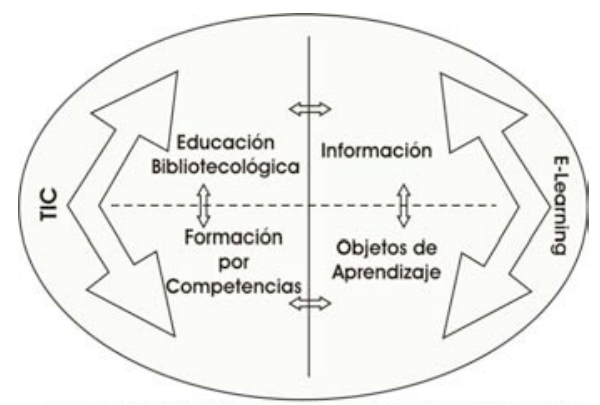

Figura 6. Educación bibliotecológica basada en AO.

Para organizar las actividades de aprendizaje del alumno y al mismo tiempo garantizar una secuencia lógica en los programas de formación, propiciando un acercamiento progresivo desde la situación inicial del alumno hasta los objetivos que persigue el plan de estudios de la institución, será necesario diseñar objetos de aprendizaje correspondientes con los objetivos educativos, las actividades y las competencias.

Al diseñar programas de educación bibliotecológica basados en un esquema de objetos de aprendizaje, vale la pena tener en cuenta las palabras de Estivill (2006), quien sostiene que:

las universidades que forman bibliotecarios deben enfocar sus currículos para poder desarrollar profesionales altamente competentes, adaptar sus conocimientos técnicos y capacidades personales al mercado de trabajo. Además, es importante que las universidades acerquen a sus estudiantes al mercado laboral a través de prácticas y trabajos en terreno, para poder fomentar el desarrollo de habilidades técnicas, así como incentivar el trabajo de equipos, la cooperación y la orientación al logro.

Reafirmando lo anterior, Pirela (2006), sostiene que el modelo curricular por competencias demanda reorientar las prácticas educativas, además de revisar la selección y organización de los contenidos y actividades de enseñanza. Es decir, se hace necesario desmontar el esquema de transmisión unilateral de conocimientos, así como superar el aprendizaje memorístico, para abrir paso a espacios de interpretación, reflexión, experimentación y debate sobre los temas que se discutan, para resignificarlos $y$ reapropiarlos a nivel individual y colectivo. Por consiguiente, el carácter acabado de los contenidos debe ser modificado para entenderlos como constructos en permanente revisión y adecuación. Todo lo cual puede ser extrapolado con el diseño de objetos de aprendizaje.

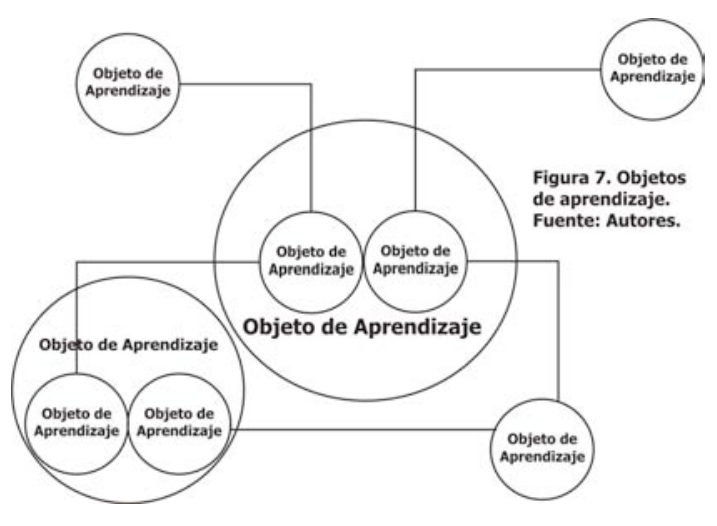

Figura 7. Objetos de aprendizaje.

\section{Consideraciones finales}

En el contexto actual las TIC no tienen confines previsibles. Su aplicación en el ámbito educativo ha contribuido al desarrollo y disponibilidad de materiales didácticos, así como de medios y escenarios en los cuales al alumno se convierte en el personaje principal del proceso, priorizando sus necesidades, competencias, capacidades, habilidades, etc.

Los nuevos tiempos necesitan pensamientos modernos e inéditos acercamientos. En un primer momento, los cambios se perfilan inadvertidos para después hacerse conscientes. Así, la nueva disposición de este cambio está teniendo efectos no sólo en el contenido de los conocimientos, sino en el modo en que éstos están organizados.

La formación de los profesionales requiere de una nueva visión para entender y transmitir la Bibliotecología en términos de conocimientos disciplinares-teóricos puestos en operación, que les permita gestionar los procesos de construcción y distribución de estos conocimientos. Esta forma de entender la disciplina tiene que ver con definir conocimientos nucleares y conocimientos periféricos a los conceptos, y entre ellos definir sus relaciones y dependencias. Obtendríamos finalmente una malla compleja de interacciones entre contenidos de distintos tipos.

Para potencializar las bondades de los entornos de aprendizaje soportados por TIC, se acude a las soluciones e innovaciones ocurridas en diversas áreas del conocimiento. Con el crecimiento de Internet y las innovaciones que se presentan, ha sido posible desarrollar la idea de 
ensamblar pequeñas unidades de información, sustentadas en la teoría del diseño instruccional y gracias a sus características de reusabilidad, capacidad generativa, adaptabilidad y escalabilidad. Por ello, los OA resultan ser el puente de comunicación en el que el alumno se apoya para integrar, seleccionar e interactuar con el conocimiento presentado, con la finalidad de desarrollar su aprendizaje.

Consideramos que los OA son entidades digitales cuya característica principal es fungir como herramientas pedagógicas que facilitan y proporcionan un aprendizaje significativo; y qe la tendencia es fomentar el autoaprendizaje a través de una metodología dinámica e investigativa encaminada a la comunidad bibliotecológica, demostrando que el aprendizaje desarrollado se centrará tomando en cuenta las necesidades específicas del usuario a quien va dirigido.

El diseñar OA para la disciplina supone entonces una visión estratégica que pueda ir más allá de la elaboración de recursos por parte del docente. Requiere de un plan de diseño en el que se opere con un grupo interdisciplinario para desarrollar patrones de objetos de aprendizaje con tipos de actividades aplicables y transversales a numerosos cursos. Asimismo las instituciones educativas necesitan plantear esta propuesta porque pueden disponer de repositorios, los cuales podrían estar orientados por un ánimo de colaboración, pues los OA pueden ser sujetos de intercambio. Además, están encaminados a la capacitación basada en habilidades y competencias, permitiendo construir un programa de capacitación exactamente a la medida del perfil del alumno (Wiley, 2001).

Resulta evidente que enseñar y aprender desde la perspectiva de los objetos de aprendizaje exige nuevas formas pedagógicas, dado que los diseños educativos así como los desarrollos y procesos de aprendizaje han de plantearse de manera muy diferente (Hodgins, 2000).

En este escenario, los objetos de aprendizaje son recursos que pudieran potenciar y conciliar las capacidades de manejo de información con las necesidades de conocimiento que permanentemente precisan los profesionales de la bibliotecología. Los objetos de aprendizaje no son sólo la extensión moderna del E-learning; van mucho más allá para convertirse en el elemento que, gracias a su versatilidad, ofrece la posibilidad de completar y mantener la formación de los profesionales de la información, con una optimización de recursos.

Finalmente, creemos que es necesario adoptar el enfoque de formación por competencias en la disciplina, toda vez que dotará al alumno con diversos elementos organizados que le permitirán decidir la relevancia del conocimiento que pretende aprehender, así como los objetos de aprendizaje a utilizar, los cuales mediarán su proceso educativo. Como señala Suárez (2002) el conocimiento es el vehículo que transporta la competencia, y la inteligencia es el lubricante que facilita el progreso; ambas cuestiones condicionan los niveles y las prestaciones del producto final resultante.

\section{Notas}

(1) Estudios realizados en la Universidad de Harvard por David McClelland ponían de manifiesto que los expedientes académicos, los conocimientos adquiridos y los tests de inteligencia no proporcionaban la información suficiente para determinar de manera fiable la idoneidad de las personas a los diferentes puestos de trabajo, ni para presagiar niveles o carreras profesionales de éxito. Es en este contexto que adopta el término Competency, como una nueva referencia, una unidad de medida alternativa o complementaria para identificar variables que predijeran el rendimiento laboral: lo que las personas deben ser capaces de llevar a cabo, asociadas a las funciones, es decir, su competencia profesional; en segundo lugar, las tareas y obligaciones propias de los puestos $u$ ocupaciones, y el tercer elemento está constituido por la eficiencia en el rendimiento profesional, medido en términos de adecuación y logros.

(2) En su obra Human Capital: a Theoretical and empirical analysis, with special reference to education plantea que una fuerza de trabajo bien entrenada es un importante activo para un país, destacando de igual forma que el capital humano es importante porque la productividad de las economías modernas se basa en la creación, difusión y utilización del conocimiento.

(3) Las instituciones que imparten estudios profesionales y de posgrado en el área bibliotecológica en México son: UNAM, Facultad de Filosofía y Letras (Licenciatura, Maestría y Doctorado); Escuela Nacional de Biblioteconomía y Archivonomía, SEP (Licenciatura); Universidad Autónoma de San Luis Potosí, Facultad de Bibliotecología e Información (Licenciatura); Universidad Autónoma de Nuevo León, Fac. de Filosofía y Letras (Licenciatura); Universidad Autónoma del Estado de México, Lic. en Ciencias de la Información Documental; Universidad Autónoma de Chiapas, Lic. en Bibliotecología; Universidad Autónoma de Guadalajara, Lic. en Ciencias de la Información; Universidad Autónoma de Chihuahua, Lic. en Ciencias de la Información; El Colegio de México, A. C., Biblioteca Daniel Cosío Villegas, Maestría en Bibliotecología; ITESM, Maestría en Ciencias de la Información y Administración del Conocimiento; Universidad Autónoma Metropolitana Xochimilco, Maestría en Gestión y Uso de la Información. 


\section{Referencias}

Alves, Lynn; Souza, Antonio Carlos (2005). Objetos digitaes de aprendizagem: tecnología e educação. // Revista de Faeeba. 14:23 (2005) 41-50

Banks, B. (2001). Learning Theory and Learning Objects. <http://www.fdlearning.com/html/company/papers/elearnContentPaper.pdf> (2007-02-21)

Banks, B.; McGrath, Kyle (2003). E-Learning Content Advisory Paper. <http://www.fdlearning.com/html/> (2007-0213)

Bower, G.H.; Hilgard, Ernest R. (1989). Teorías del aprendizaje. México: Trillas, 1989.

Bunk, G. P. (1994). Teaching competence in initial and continuing vocational training in the Federal Republic of Germany. // Vocational Training European Journal. 1 (1994) 8-14.

Chan Núñez, Maria Elena (2002). Objetos de aprendizaje: una herramienta para la innovación educativa. // Apertura 2 (Dic. 2002) 3-9

Debons, A.; Horne, E.; Cronenweth, S. (1998). Information science: an integrated view. Boston: G.K. Hall, 1998.

The definition and selection of key competencies: executive summary. <http://www.oecd.org/dataoecd/47/61/350703 67.pdf> (2007-03-11)

Delclaux, Isidoro; Seoane, Julio (1982). Psicología cognitiva y procesamiento de la información: teoría, investigación y aplicaciones. Madrid: Ediciones Pirámide, 1982.

Draxler, A (2004). ¿Qué es el e-learning: un nuevo paradigma o un nuevo juguete? [en línea]. <http://elear ningeuropa.info> (2007-01-11)

Ellis, David (1992a). The physical and cognitive paradigms in Information Retrieval Research. // Journal of Documentation. 48:1 (March 1992) 45-46.

Garduño Vera, Roberto (2006). Objetos de aprendizaje en la educación virtual: una aproximación en bibliotecología. // Investigación Bibliotecológica: archivonomía, biblioteconomía e información. 20:41 (Jul.-Dic. 2006) 35-55

Gerard, R.; Whitlock, G. (1969). Shaping the mind: computers in education. // Training \& Development Journal. 23:2 (Feb.1969) 1-4.

Holmberg, B. (1985). Educación a distancia: situación y perspectivas. Argentina: Kapelusz, 1985.

Johnson, S. (1992). Librarians teaching technology with technology. // Information Retrieval and Library Automation. 11-5.

Karisiddappa, CR. (2004). Plan de estudios en biblioteconomía en vías de desarrollo World. // Library and Information Congress (70: 2004: Buenos Aires, Arg.) $<$ http://www.ifla.org/lV/ ifla70/prog04.htm> (2007-05-11)

Line, M.B. (1998). Le métier de bibliothécaire: un ensemble de pratiques confuses et discontinúes. // Bulletin des bibliothéques de France 43:2 44-48 <www.enssib.fr/bbf> (2007-01-16)

López Camps, Jordi; Leal Fernández, Isaura (2002). Como aprender en la sociedad del conocimiento. Barcelona: Gestion 2000, 2002.

Mababu M. R. (2003). Entornos virtuales de aprendizaje. <http://mecd.es/2/richard ind.html> (2007-04-11)

Markey, Karen (1990). Keyword searching in an online catalog enhanced with a library classification. // Bengtson, Betty G.; Hill, Janet Swan (eds.). Classification of library materials: current and future potential for providing access. New York: Neal-Shuman Publishers, 1990. 99-125.

Merrill, M.D., Li, Z. \& Jones, M. (1991). Instructional transaction theory: an introduction. // Educational Technology. 31:6 (1991) 7-12.

Morales Campos, Estela (2003). Bibliotecología y sociedad. // Morales Campos, Estela; Ríos Ortega, Jaime (comp.)
Mesa redonda. Bibliotecología, educación y sociedad. México: UNAM, CUIB, 2003. 1-11.

Muñoz Arteaga, Jaime; Álvarez R., F.J.; Osorio U., B. (2006). Objetos de aprendizaje integrados a un sistema de gestión de aprendizaje. // Apertura. 6:3 (Abr. 2006) 109-117.

Navarro Cendejas, José; Ramírez Anaya, Luis F. (2005). Objetos de aprendizaje. Formación de autores con el modelo redes de objetos. México: UDG Virtual, 2005.

Oakes, K. (2002). An objective view of LO. // T+D 56:2 1-3.

Pirela Morillo, Johann (2006). Tendencias y perspectivas para la enseñanza de la bibliotecología en la cibersociedad. Martínez A., F. Felipe; Calva G. J. José (comp.) Memoria del XXIII Coloquio de Investigación Bibliotecológica y de la Información. México: UNAM, CUIB, 2006. 85-108

Pirela Morillo, Johann; Peña Vera, Tania (2005). Nuevos desafíos para la formación del profesional de la información frente al surgimiento de la cibersociedad: un enfoque de competencias. // Investigación Bibliotecológica: archivonomía, biblioteconomía e información. 19:38 (Jul.-Dic. 2005) 118-139.

Ramírez Ortega, Alfonso (2006). De conceptos, sitios y funcionalidades: en las tecnologías educativas. // Innovación educativa. 20 (2006) 45-69.

Ríos Ortega, Jaime (2007). La teoría en la educación bibliotecológica. Directrices básicas para su enseñanza. // Investigación Bibliotecológica: archivonomía, biblioteconomía e información. 21:42 (Ene.-Jun. 2007) 118-139.

Tobón Tobón, Sergio (2004). Formación basada en competencias. Bogotá: Ecoe Ediciones, 2004.

Salas Zapata, Walter A. (2005). Formación de competencias en la educación superior una aproximación conceptual a propósito del caso colombiano. // Revista Iberoamericana de Educación 36:9 (2005). <http://www.rieoei.org/ deloslectores/1036Salas.PDF> (2007-03-15)

Santacruz Valencia, L.P. (2005). Automatización de los procesos para la generación, ensamblaje y reutilización de objetos de aprendizaje. Tesis Doctoral. España: U. Carlos III de Madrid, 2005.

Simone, Raffaele (2001). La tercera fase: formas de aprender que estamos perdiendo. Madrid: Santillana, 2001.

Smith Nash, Susan (2005). Learning objects, learning object repositories, and learning Theory: preliminary best practices for online courses. // Interdisciplinary Journal of Knowledge and Learning Objects. 1 (2005) 217-228.

Smith, Ph. J.; Beghtol, C.; Fidel, R.; Kwasnik, B. H. (eds.) (1993). Proceedings of the 4th ASIS SIG/CR Classification Research Workshop: Columbus, OH, 1993. Silver Spring, MD.: American Society for Information Science, 1993.

Valdez Coiro, I. S.(2006). El enfoque de competencias en la virtualidad educativa. // Apertura 6:4 (2006) 23-32

Wiley, D. The instructional use of learning objects: online. <http://www.reusability.org/readwww.rieo ei.org/deloslectores/1036Salas.PDF>(2007-03-22)

Wilson, H.A. Computer-Assisted: A book of Readings. New York: Academic Press.

Zambrano Leal, A. (2006). Tres tipos de saber del profesor y competencias: una relación compleja. // Educere. 10:3 (2006). 221-229.

Zapata Ros, Miguel (2005). Secuenciación de contenidos y objetos de aprendizaje. // Revista de Educación a Distancia 4:11 <http://redalyc.uaemex.mx/redalyc/src/ inicio/ArtPdfRed.jsp?iCve=54709510> (2007-03-15) 